\title{
Determination of the strong coupling at NNLO from jet production in DIS
}

\section{Daniel Britzger* on behalf of the collaboration ${ }^{\dagger}$}

DESY, Notkestr. 85, 22607 Hamburg, Germany

E-mail: daniel.britzger@desy.de

A first determination of the strong coupling $\alpha_{s}\left(m_{Z}\right)$ in next-to-next-to leading order (NNLO) from inclusive jet and dijet production cross sections in deep-inelastic scattering at HERA is presented [四]. Data collected by the H1 experiment in the years 1995 to 2007 covering the range of momentum transfer $5.5<Q^{2}<15000 \mathrm{GeV}^{2}$ and jet transverse momenta $P_{\mathrm{T}}^{\text {jet }}>4.5 \mathrm{GeV}$ are explored. The strong coupling is determined in a fit to inclusive jet and dijet data to $\alpha_{s}\left(m_{Z}\right)=0.1157(6)_{\exp }\left({ }_{-26}^{+31}\right)_{\text {theo }}$. Further studies on the phenomenological application of the new NNLO calculations and on fits to the individual data sets are presented. The running of the strong coupling is probed in a single experiment over one order of magnitude in the remormalisation scale and consistency with the QCD expectations is found.

XXV International Workshop on Deep Inelastic Scattering and Related Topics

3-7 April, 2017

University of Birmingham, $U K$

\footnotetext{
*Speaker.

${ }^{\dagger}$ Work performed by the H1 Collaboration together with V. Bertone, J. Currie, C. Gwenlan, T. Gehrmann, A. Huss,
} J. Niehues and M. Sutton 


\section{Introduction}

The strong coupling constant is one of the least known parameters of the Standard Model (SM) and a precise knowledge is of crucial importance for precision physics and searches for physics beyond the SM at the LHC. Cross sections for jet production in deep-inelastic electron-proton scattering (DIS) are directly sensitive to the strong coupling constant $\alpha_{s}\left(m_{Z}\right)$ already in leading order in perturbative QCD (pQCD) as these measurements are performed in the Breit frame of reference. The cross section calculations are performed in next-to-next-to-leading order (NNLO)

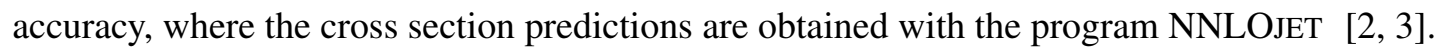

\section{Methodology}

Cross sections for jet production in $e p$ collisions have been measured by the $\mathrm{H} 1$ experiment at HERA at different center-of-mass energies and for different kinematic regions, and we consider inclusive jet and dijet cross sections taken during the years 1995 to 2007 [四, [1, 团, 四, 四]. Consistent to all data sets, jets are defined using the $k_{t}$ jet-algorithm with a parameter of $R=1$, and jets are required to be contained in the pseudorapidity range $-1<\eta_{\mathrm{lab}}^{\mathrm{jet}}<2.5$ in the laboratory frame. For the selected data, inclusive jet cross sections have been measured double-differentially as a function of the photon virtuality $Q^{2}$ and jet transverse momentum $P_{\mathrm{T}}^{\mathrm{jet}}$, and dijet cross sections as a function of $Q^{2}$ and the average transverse momentum of the two hardest jets, $\left\langle P_{\mathrm{T}}\right\rangle$. A brief summary of the employed measurements and the kinematic range of the observables is given in table $\mathbb{W}$. The data

\begin{tabular}{|c|c|c|c|c|c|}
\hline $\begin{array}{l}\text { Data set } \\
\text { [Ref.] }\end{array}$ & $\begin{array}{c}\sqrt{s} \\
{[\mathrm{GeV}]}\end{array}$ & $\begin{array}{l}\text { int. } \mathcal{L} \\
{\left[\mathrm{pb}^{-1}\right]}\end{array}$ & $\begin{array}{l}\text { DIS kinematic } \\
\text { range }\end{array}$ & Inclusive jets & $\begin{array}{c}\text { Dijets } \\
n_{\text {jets }} \geq 2\end{array}$ \\
\hline $300 \mathrm{GeV}$ [䧃] & 300 & 33 & $150<Q^{2}<5000 \mathrm{GeV}^{2}$ & $7<P_{\mathrm{T}}^{\mathrm{jet}}<50 \mathrm{GeV}$ & $8.5<\left\langle P_{\mathrm{T}}\right\rangle<35 \mathrm{GeV}$ \\
\hline HERA-I [回] & 319 & 43.5 & $5<Q^{2}<100 \mathrm{GeV}^{2}$ & $5<P_{\mathrm{T}}^{\mathrm{jet}}<80 \mathrm{GeV}$ & $7<\left\langle P_{\mathrm{T}}\right\rangle<80 \mathrm{GeV}$ \\
\hline HERA-I [回] & 319 & 65.4 & $150<Q^{2}<15000 \mathrm{GeV}^{2}$ & $5<P_{\mathrm{T}}^{\mathrm{j} e t}<50 \mathrm{GeV}$ & - \\
\hline HERA-II ["]] & 319 & 290 & $5.5<Q^{2}<80 \mathrm{GeV}^{2}$ & $4.5<P_{\mathrm{T}}^{\mathrm{jet}}<50 \mathrm{GeV}$ & $5<\left\langle P_{\mathrm{T}}\right\rangle<50 \mathrm{GeV}$ \\
\hline 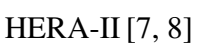 & 319 & 351 & $150<Q^{2}<15000 \mathrm{GeV}^{2}$ & $5<P_{\mathrm{T}}^{\mathrm{jet}}<50 \mathrm{GeV}$ & $7<\left\langle P_{\mathrm{T}}\right\rangle<50 \mathrm{GeV}$ \\
\hline
\end{tabular}

Table 1: Summary of the kinematic ranges of the inclusive jet and dijet data taken by the H1 experiment.

sets are present for the different data taking periods and for two $Q^{2}$-ranges, where the scattered lepton is identified in different experimental devices. In case of the dijet cross sections, regions of the phase space exhibiting an infrared sensitivity due to 'back-to-back' topologies are avoided by imposing asymmetric cuts on the transverse momenta of the two leading jets.

The predictions are calculated as a convolution of parton density functions (PDFs) and a partonic cross section. Both these components exhibit a dependence on $\alpha_{s}\left(m_{Z}\right)$ and their impact on the results are assessed below. The partonic cross section has its $\alpha_{s}$-dependence explicit as it is calculated in terms of a perturbative expansion in orders of $\alpha_{s}^{(n)}$. The $\alpha_{s}$-dependence of the PDFs is defined by the factorisation theorem and thus it is expressed by the QCD splitting kernels and the $\beta$-functions. Consequently, once a PDF is determined for a given value of $\alpha_{s}\left(m_{Z}\right)$ it can be translated to any other value of $\alpha_{s}\left(m_{Z}\right)$ by integration, which introduces the $\alpha_{s}$-dependence of the PDF. An equivalent solution to this explicit integration is obtained by evaluating the PDFs at a suitable value of $\mu_{F}$, and thus taking full benefit of the factorisation theorem [四]. The NNLO calculations 
are performed using the NNPDF3.0 PDF [Q], which was determined for a value of $\alpha_{s}\left(m_{Z}\right)=0.118$. Multiplicative correction factors are applied in order to account for non-perturbative hadronisation effects. The renormalisation and factorisation scales are chosen to be $\mu_{R}^{2}=\mu_{F}^{2}=Q^{2}+P_{\mathrm{T}}^{2}$, where $P_{\mathrm{T}}$ denotes $P_{\mathrm{T}}^{\mathrm{jet}}$ in case of inclusive jet and $\left\langle P_{\mathrm{T}}\right\rangle$ for dijet cross sections.

The value of the strong coupling constant is determined in a fit of NNLO calculations to the $\mathrm{H} 1$ jet data, where the $\alpha_{s}$-dependencies in the predictions, both in the partonic cross sections and in the PDF, are taken into account. The NNLO coefficients are stored in the fastNLO format [U]] in order to allow for a repeated calculation with different values of $\alpha_{s}\left(m_{Z}\right)$ and different PDF sets. The $\chi^{2}$-definition accounts for experimental, hadronisation and PDF uncertainties. Correlations of systematic uncertainties are accounted for and also the statistical correlations of the data are considered. Uncertainties on the resulting value of $\alpha_{s}\left(m_{Z}\right)$ due to experimental and theoretical sources are estimated. The PDF and hadronisation uncertainties are obtained by repeating the fit with these uncertainties excluded in the fit and comparing the resulting fit uncertainty. The scale uncertainty is estimated by repeating the fit with scale factors of 0.5 and 2 . The 'PDFSet' uncertainty is obtained as half of the maximum difference of the results from fits using alternatively the ABMP, CT14, HERAPDF2.0, MMHT or NNPDF3.0 PDF set, and the ' $\mathrm{PDF} \alpha_{s}$ ' uncertainty is estimated as half of the difference of the results obtained from fits using PDFs which were determined with $\alpha_{s}\left(m_{Z}\right)$-values differing by 0.004 .

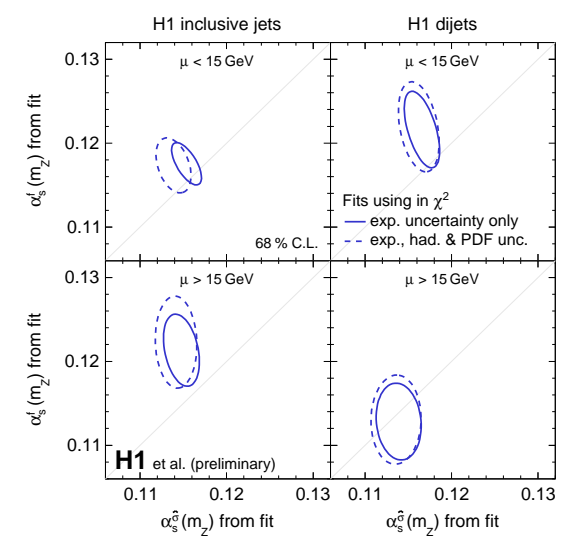

Figure 1: Contours at a confidence level of $68 \%$ for fits where the two appearances of $\alpha_{s}\left(m_{Z}\right)$ in the cross section calculation are identified separately. The upper and lower pads show results from data points with $\mu_{R}$ smaller or greater $15 \mathrm{GeV}$. The dashed contours indicate fits where the PDF uncertainty is not considered in the $\chi^{2}$-calculation.

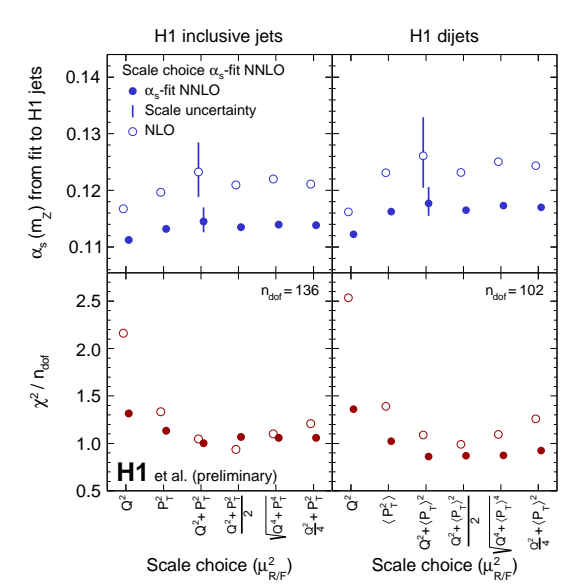

Figure 2: Values of $\alpha_{s}\left(m_{Z}\right)$ obtained from fits to inclusive jet or dijet cross sections obtained for different definitions of the renormalisation and factorisation scales. The lower pads show the values of $\chi^{2} / n_{\text {dof }}$ of the fit. The open circles display results obtained using NLO matrix elements. The vertical error bars indicate the scale uncertatinty.

\section{Results}

The sensitivity of the data to $\alpha_{s}\left(m_{Z}\right)$ is studied in fits with two free parameters representing the two $\alpha_{s}$ contributions to the calculation, if those are separately identified, i.e. the one of the 
PDFs, $\alpha_{s}^{f}\left(m_{Z}\right)$, and the one of the hard coefficients, $\alpha_{s}^{\hat{\sigma}}\left(m_{Z}\right)$. The fits are performed using inclusive jet or dijet cross section measurements, with data points below or above the renormalisation value of $15 \mathrm{GeV}$. The contours displaying the $68 \%$ confidence level of the fitted results are displayed in figureW. It is found that the fitted $\alpha_{s}\left(m_{Z}\right)$ values determined are consistent and the sensitivity to $\alpha_{s}\left(m_{Z}\right)$ of the hard coefficients outperforms the one of the PDF. The two fit parameters are negatively correlated, resulting in an increased sensitivity for the final results on $\alpha_{s}\left(m_{Z}\right)$.

Fits are performed employing alternative definitions for the renormalisation and factorisation scales. The resulting $\alpha_{s}$-values and related values of $\chi^{2} / n_{\text {dof }}$ for the individual fits are displayed in figure $\square$ for fits to inclusive jet and to dijet cross sections. The results obtained with alternative scale choices are typically covered by the scale uncertainty. Choosing $\mu_{R}^{2}=\mu_{F}^{2}=Q^{2}$ is disfavored, presumably because this scale is not sufficiently related to the dynamics of jet production. The fits are also repeated with hard coefficients calculated in NLO accuracy only. These calculations exhibit significantly higher values of $\chi^{2} / n_{\text {dof }}$. In addition the scale choice has a higher impact on the result and on the $\chi^{2}$-values. These observations confirm the improved perturbative convergence of the NNLO calculations as compared to NLO accuracy.

The values for $\alpha_{s}\left(m_{Z}\right)$ obtained from fits to the individual data sets are displayed in figure $B$ and compared to the world average value of $\alpha_{s}\left(m_{Z}\right)=0.1181 \pm 0.0011$ [U]]. The results obtained when using only inclusive jet data or only dijet data are also shown. An overall reasonable consistency between the results from the individual data sets is found, which is further confirmed by the $\chi^{2} / n_{\text {dof }}{ }^{-}$ values around unity of the fits to all of the inclusive jet or dijet data (c.f. also fig. (2).

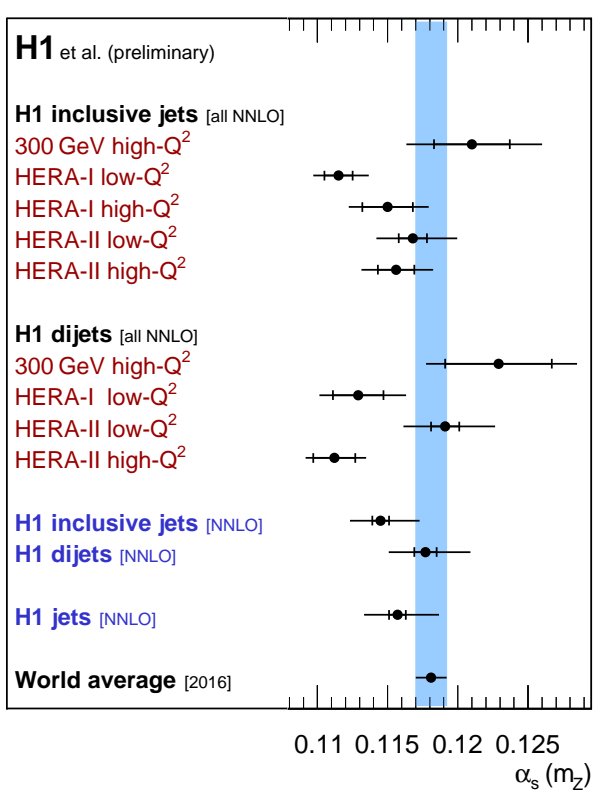

Figure 3: Summary of the values of $\alpha_{s}\left(m_{Z}\right)$ obtained from fits to the individual data sets and from fits to multiple data sets. The inner errors bars indicate the experimental uncertainty and the outer error bars the total uncertainty.

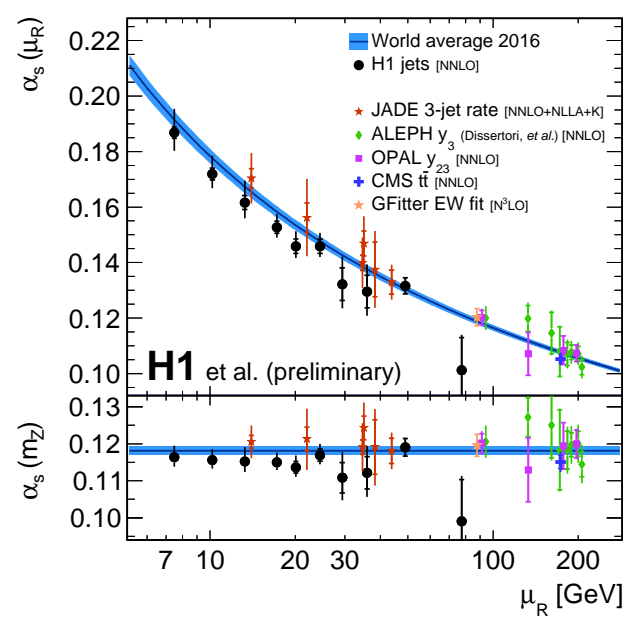

Figure 4: Values of $\alpha_{s}\left(m_{Z}\right)$ obtained from fits to 'H1 jets' data points with similar values of $\mu_{R}$ (full circles) in comparison to values from other experiments and processes, where all values are obtained at least in NNLO accuracy. The fitted values of $\alpha_{s}\left(m_{Z}\right)$ are translated to $\alpha_{s}\left(\mu_{R}\right)$ using the solution of the QCD renormalisation group equation as they also enter the calculations. The inner error bars display the experimental uncertainties and the outer error bars indicate the total uncertainties. 
A fit to all $\mathrm{H} 1$ jet cross section data (denoted ' $\mathrm{H} 1$ jets'), where only HERA-I dijet cross sections are excluded from the fit because their statistical correlation to the inclusive jets are not precisely known, yields a value of $\chi^{2} / n_{\text {dof }}=1.03$ for 203 data points and the value of the strong coupling constant $\alpha_{s}\left(m_{Z}\right)$ is determined to

$$
\alpha_{s}\left(m_{Z}\right)=0.1157(6)_{\mathrm{exp}}(3)_{\mathrm{had}}(6)_{\mathrm{PDF}}(12)_{\mathrm{PDF} \alpha_{\mathrm{s}}}(2)_{\mathrm{PDFset}}\left({ }_{-21}^{+27}\right)_{\mathrm{scale}} .
$$

This is consistent with the world average and with fits of the individual data sets. The fit to all data exhibits a significantly reduced experimental uncertainty as compared to the results obtained from the single data sets.

The running of the strong coupling constant as a function of the renormalisation scale $\mu_{R}$, is studied by repeating the fit for groups of data points with comparable values of $\mu_{R}$. The resulting values of $\alpha_{s}\left(m_{Z}\right)$, displayed at a representative value $\mu_{R}$ for the given range, and the respective value of $\alpha_{s}\left(\mu_{R}\right)$, are displayed in figure $\theta$. The results confirm the expectations from the QCD renormalisation group equation within the accessible range in $\mu_{R}$ of approximately 7 to $90 \mathrm{GeV}$. The $\alpha_{s}$-values are also compared to $\alpha_{s}$-determinations at NNLO in other reactions at similar scales and consistency with other extractions is found.

\section{Summary and conclusion}

The strong coupling constant is determined in a fit of next-to-next-to-leading order (NNLO) QCD predictions to inclusive jet and dijet cross section measurements by $\mathrm{H} 1$. The value at the mass of the $Z$-boson is determined to $\alpha_{s}\left(m_{Z}\right)=0.1157(6)_{\exp }\left({ }_{-26}^{+31}\right)_{\text {theo }}$, which is consistent with the world average value. The running of the strong coupling constant is probed over one order of magnitude and consistency with the QCD expectations is found. The new NNLO calculations reduce significantly the dominating theoretical uncertainty in comparison to previously employed NLO calculations. The experimental uncertainty is reduced by considering the entire set of inclusive jet and dijet cross section measurements by the $\mathrm{H} 1$ experiment.

\section{References}

[1] H1 Collaboration and V. Bertone et al., H1 preliminary report, H1prelim-17-031 (2017).

[2] J. Currie, T. Gehrmann, and J. Niehues, Phys. Rev. Lett. 117 (2016) 042001, arxiv:1606.03991.

[3] J. Currie, T. Gehrmann, A. Huss, and J. Niehues, arxiv:1703.05977.

[4] H1 Collaboration, C. Adloff et al., Eur. Phys. J. C19 (2001) 289, arXiv:hep-ex/0010054.

[5] H1 Collaboration, F. D. Aaron et al., Eur. Phys. J. C67(2010)], arX1v:0911.5678.

[6] H1 Collaboration, A. Aktas et al., Phys. Lett. B653 (2007) 134, arXiv:0706.3722.

[7] H1 Collaboration, V. Andreev et al., arXiv:1611.03421.

[8] H1 Collaboration, V. Andreev et al., Eur. Phys. J. C75 (2015) 65, arXiv: 1406.4709.

[9] NNPDF Collaboration, R. D. Ball et al., VHEP 04(2015) 040, arXiv: 1410.8849.

[10] D. Britzger et al., Conf. Proc. C12-03-26.1 (2012) 217, arXiv:1208.3641.

[11] Particle Data Group Collaboration, C. Patrignani et al., Chin. Phys. C40 (2016) 100001. 